\title{
Estimating the lifetime of marine concrete
}

\section{Barry McDonald ${ }^{1} \quad$ Graeme Hastie $^{2} \quad$ Graeme Wake $^{3}$}

(Received 2 March 2010; revised 8 June 2010)

\begin{abstract}
When concrete is exposed to salt water, chloride ions diffuse into the concrete and cause the reinforcing steel to rust. Industry standards set the effective lifetime of concrete as the time at which the chloride ion concentration at the steel surface exceeds some threshold (for example, $0.5 \%$ of binder). The problem is to find a lower bound on the time, for every depth of concrete between the sea and the underlying steel, so that one can state with, say, $95 \%$ confidence that the concrete lifetime exceeds that bound. Estimation is complicated by the fact that there is only five years of data and we predict fifty or more years into the future. The solution set forth has two steps. First, a parametric model is proposed for the concentration field, based on Fick's law for diffusion, but modified to allow for a non-constant diffusion coefficient. Within the concrete industry the usual modification is an approximate method, but an exact solution is derived in an appendix. Both the approximate and exact methods are tested on data. Second, given a depth of concrete, lower confidence bounds on lifetime are estimated by smoothing a parametric bootstrap of the concentration field over a grid in space-time using interpolation. Some consistent differences emerge between the exact
\end{abstract}

http://anziamj . austms.org.au/ojs/index.php/ANZIAMJ/article/view/2426 gives this article, (c) Austral. Mathematical Soc. 2010. Published June 29, 2010. ISSN 1446-8735. (Print two pages per sheet of paper.) 
and approximate results, which may have implications for the concrete industry.

\section{Contents}

1 Introduction

C410

2 Parametric model for the concentration field

3 Estimating model parameters and errors

4 Obtaining confidence bounds by simulation

C418

5 Discussion

A Proof of exact formula

References

\section{Introduction}

Marine grade concrete is used in many civil engineering projects such as bridges and wharves. However, chloride ions from salt water gradually diffuse into the concrete. Once these ions reach the reinforcing steel in sufficient concentration, rust weakens the steel, which can result in catastrophic failure of the concrete [1].

Rather than measuring the useful lifetime of concrete by the actual time to failure, industry standards define the effective lifetime of marine concrete as the time $t$ at which the concentration of chloride ions at the steel exceeds some level (such as $0.4 \%$ or $0.5 \%$ of binder). The depth of concrete between the steel and concrete/water interface is called the 'cover', $x$. Let $C(x, t)$ be 
the chloride ion concentration at depth $x$ and time $t$. The task addressed in this article is that of estimating a lower confidence bound time $T$ such that one can be $95 \%$ confident that the concrete lifetime exceeds T. Putting it another way, we want to be $95 \%$ confident that the concentration satisfies $\mathrm{C}(x, t) \leqslant 0.5 \%$ of binder for time $t \leqslant \mathrm{~T}$.

For the type of concrete examined in our study, we anticipate that $T$ would be in the range 50-150 years when $x=40 \mathrm{~mm}$. However, only five years of data on $\mathrm{C}(x, t)$ were available for this type of concrete, for various combinations of $x$ and $t$. It is therefore necessary to extrapolate using a parametric model for the concentration $\mathrm{C}(x, t)$, and then use simulation to obtain confidence bounds $\mathrm{T}$.

Section 2 describes the deterministic model for $\mathrm{C}(x, t)$ in common use by the concrete industry, as well as a modified version based on further assumptions. Section 3 introduces the data used in our study, including a discussion of the uncertainty (errors) for the parameter estimates. In Section 4, values of $\mathrm{C}(\mathrm{x}, \mathrm{t})$ are simulated based on the parameter estimates and their error distributions. In this way the proportion of times that $C(x, t) \leqslant 0.5 \%$ (say) of binder are estimated. After some smoothing, interpolation estimates the confidence bound $\mathrm{T}$ for any $\mathrm{x}$, (for example such that there is $95 \%$ confidence that the lifetime exceeds T). Section 5 concludes the study.

\section{Parametric model for the concentration field}

Industry models typically start with Fick's Second Law of Diffusion, which is a differential equation for the rate of change in concentration:

$$
\frac{\partial C}{\partial t}=D \frac{\partial^{2} C}{\partial x^{2}}
$$


where $\mathrm{D}$ is a constant coefficient of diffusion for the specific type of the concrete. The differential equation has the well known solution

$$
C(x, t)=C_{i}+\left(C_{s n}-C_{i}\right) \operatorname{erfc}\left(\frac{x}{2 \sqrt{D t}}\right),
$$

where $C_{i}$ is the initial (background) ion concentration, $C_{s n}$ is the notional surface concentration, $x$ is the depth in $\mathrm{mm}$ and $\mathrm{t}$ is the time in seconds. The complementary error function

$$
\operatorname{erfc}(z)=\frac{2}{\sqrt{\pi}} \int_{z}^{\infty} e^{-t^{2}} d t=2[1-\Phi(\sqrt{2} z)],
$$

where $\Phi(\cdot)$ is the cumulative distribution function of the standard Normal distribution.

However, it is known that $\mathrm{D}$ is not constant for concrete. Industry experience suggests the model $D(t)=a t^{n}$ where $-1<n \leqslant 0$. The Fick formula (2) is therefore commonly modified [3] to

$$
C(x, t)=C_{i}+\left(C_{s n}-C_{i}\right) \operatorname{erfc}\left\{\frac{x}{2 \sqrt{\left[D_{c a\left(t_{m}\right)}\left(t / t_{m}\right)^{n}\right] t}}\right\},
$$

where $D_{c a\left(t_{m}\right)}$ is the apparent Chloride diffusion coefficient at some fixed base time $t_{m}$. The term in brackets is a rewritten form of $D(t)=a t^{n}$. The formula (4) appears to be a de facto industry standard, although other suggestions have been made $[2,4]$. However, the field (4) is no longer strictly speaking a solution to Fick's law since (1) becomes

$$
\frac{\partial C}{\partial t}=D(t) \frac{\partial^{2} C}{\partial x^{2}}=a t^{n} \frac{\partial^{2} C}{\partial x^{2}},
$$

which is a different differential equation. Its exact solution has been derived by Graeme Wake (see appendix) as

$$
C(x, t)=C_{i}+\left(C_{s n}-C_{i}\right) \operatorname{erfc}\left\{\frac{\sqrt{1+n} x}{2 \sqrt{\left[a t^{n}\right] t}}\right\} .
$$


Both the solutions (4) and (6) revert to the formula in (2) when $\boldsymbol{n}=0$. If $-1<\mathrm{n}<0$ the $\sqrt{1+\mathrm{n}}$ term means concentrations are higher, and so the concrete lifetimes are predicted to be shorter than under the currently used formula (4).

\section{Estimating model parameters and errors}

The present study was motivated by the availability of a finer raw material for making concrete, which was anticipated to produce concrete with longer lifetimes. A comparative study of several concrete mixes was carried out by researchers from BRANZ, the Building Research Association of New Zealand [5, the data considered in our article are from page 101, table 33, lower panel]. Two blocks of the specific marine grade concrete were placed in the splash zone in Wellington harbour. That is, the blocks were not consistently wet, but were splashed with salty water in windy weather, and the water would then evaporate in dry weather, concentrating the chloride on the surface of the concrete. After six months a core sample was drilled from each concrete block, and each sample was analysed for chloride ion concentration at pre-selected depths. The same procedure was carried out at 12 months, 18 months and 30 months. At 60 months three core samples were analysed. The observed concentrations are displayed in Figure 1. The pre-selected depths ranged from $1 \mathrm{~mm}$ to $37.5 \mathrm{~mm}$. Once the sample concentrations were consistently at or below the usual background level of cloride ions in concrete, assumed by concrete industry experience to be $0.01 \%$ of concrete or around $0.06085 \%$ of binder, then chemical analysis at deeper depths was discontinued.

The assumption $C_{i}=0.06085$ was verified for our data using Figure 2 which plots the square root of the concentration $\sqrt{C(x, t)}$ against $\sqrt{x}$, the square root of the depth. When $\mathrm{C}(\mathrm{x}, \mathrm{t})>\mathrm{C}_{i}$, the $\sqrt{\mathrm{C}(\mathrm{x}, \mathrm{t})}$ is related approximately linearly to $\sqrt{x}$, while for concentrations $<C_{i}$ the observed $\sqrt{C(x, t)}$ just varies randomly; that is, is unrelated to $\sqrt{x}$. Therefore the industry 


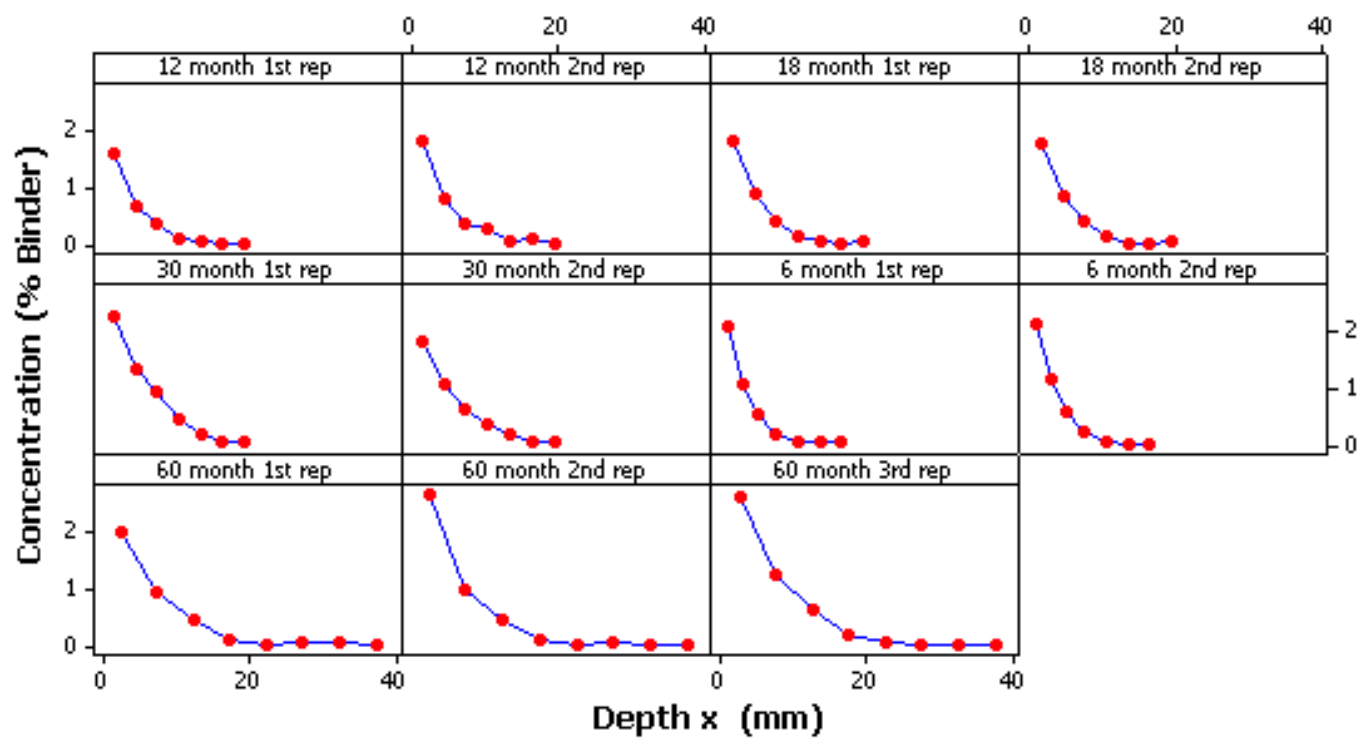

Figure 1: Chloride ion concentration versus depth within concrete, by experimental replicate, by month and replicate. 


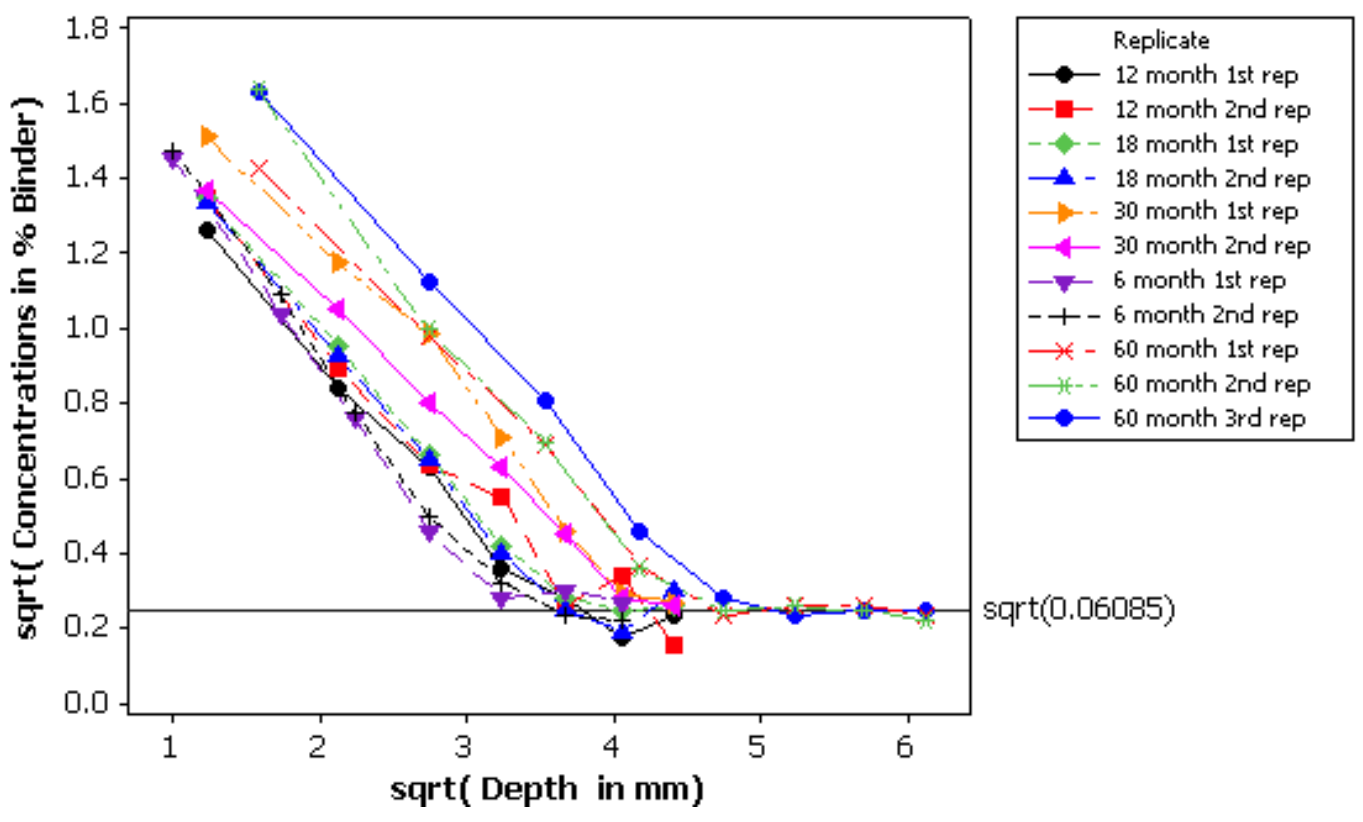

FiguRE 2: Square root concentration $\sqrt{\mathrm{C}}$ versus square root depth $\sqrt{x}$.

standard $\mathrm{C}_{i}$ was assumed, and taken to be a fixed constant, known without error. Both the industry model (4) and the exact model (6) were fitted to the data in Figure 1, and visually both provided a reasonably good fit except at the left-hand end of the curves, see Figure 3.

Fitting was performed using Solver in Excel to find $C_{s n}, a$ and $n$ to minimise the error sum of squares. There was negligible difference in the fitted values, which means that neither model could be rejected on the basis of these five years of data. Since the variance of the $\mathrm{C}(x, t)$ values seems to greater on the left than on the right of each curve, fitting was also checked by minimising a weighted sum of squares with weight proportional to $1 / \sqrt{\text { fitted values }}$ and points discarded when $\mathrm{C}(\mathrm{x}, \mathrm{t})<\mathrm{C}_{i}$. Again there was insufficient evidence that either model fitted better for these limited data.

An alternative way of representing the data shown in Figure 1 is to as- 


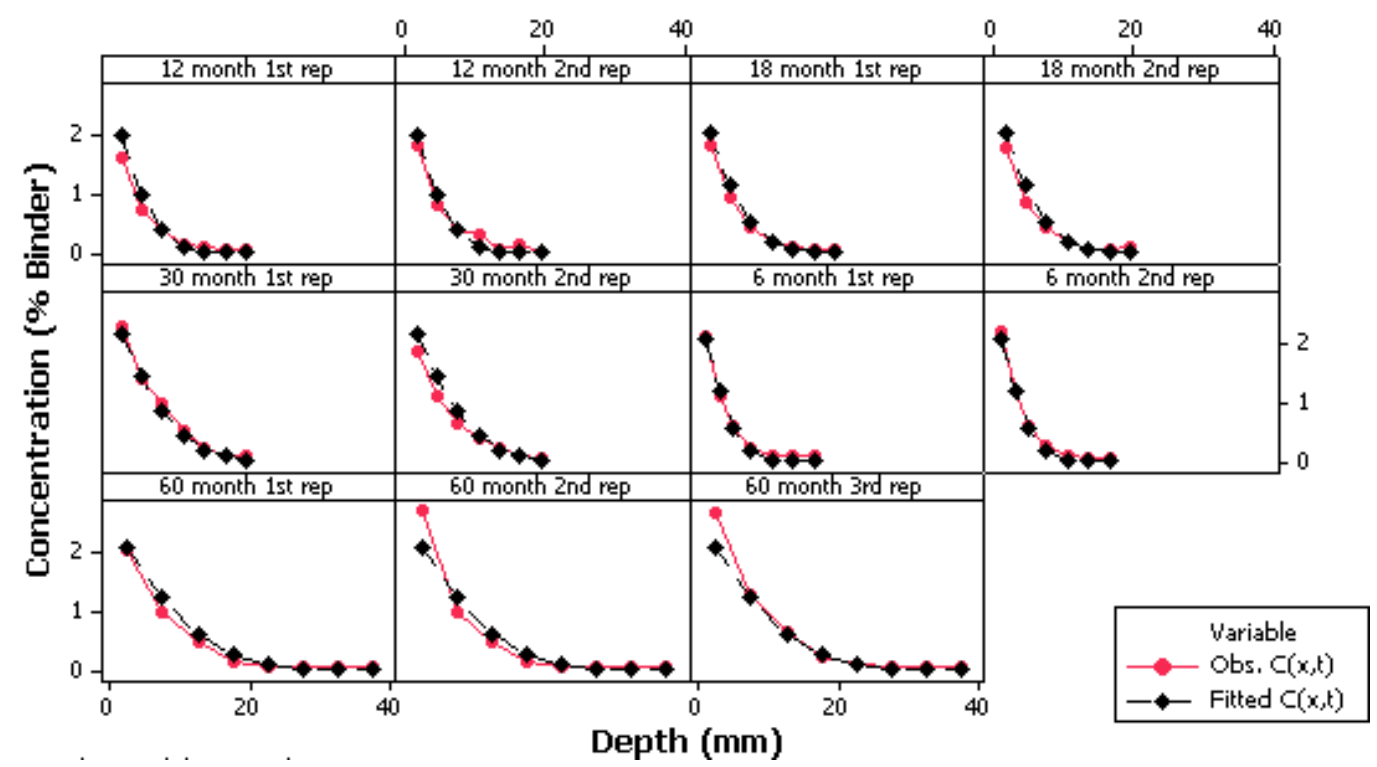

Figure 3: Observed and fitted concentrations versus depth by the usual model, plotted by month and replicate (fits using the exact model are similar). 


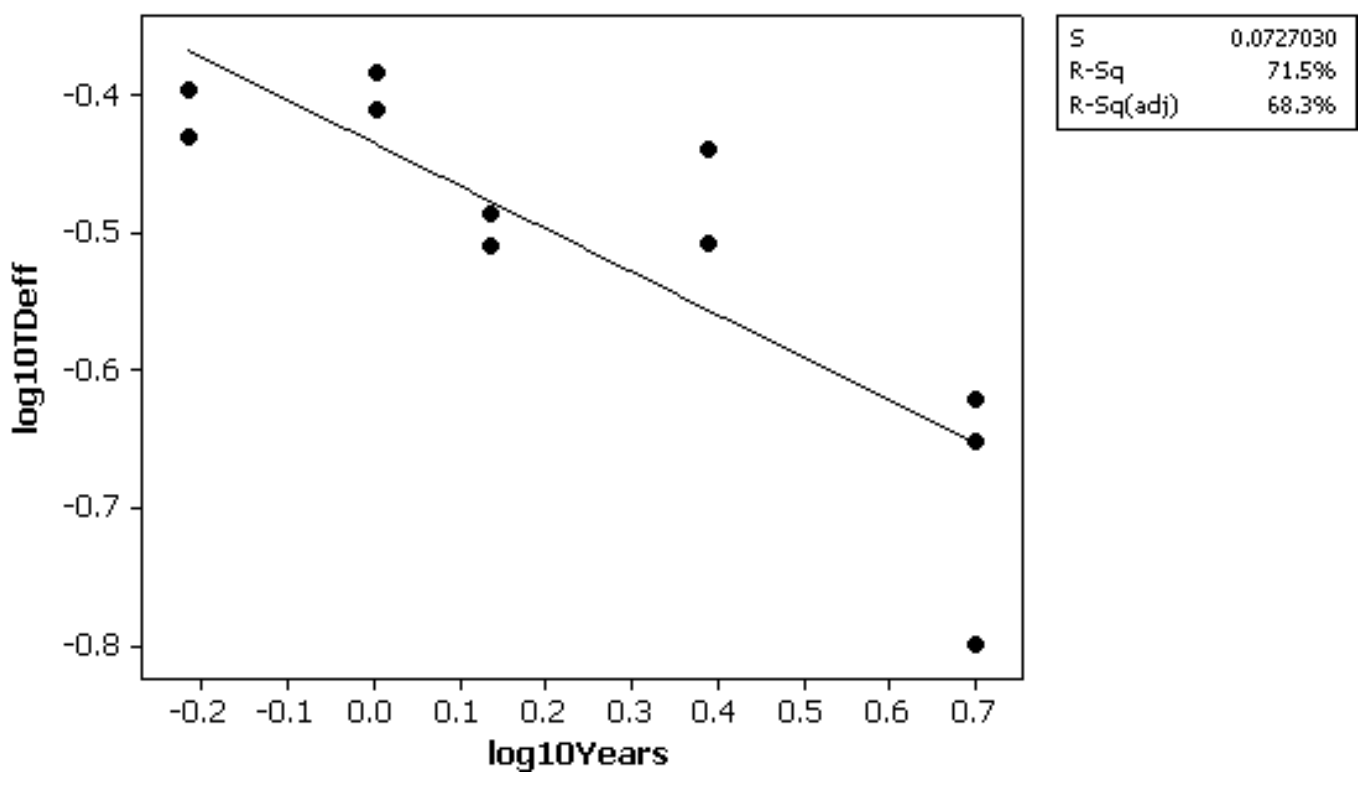

FiguRE 4: Estimated diffusion coefficients versus time, by replicate. Equation (7) gives the fitted line.

sume the surface concentration of chloride ions $C_{\text {sn }}$ can vary with time, and summarise each curve by a separate estimate of the effective coefficient of diffusion of $D_{\text {eff }}$, using the model (2). The experimental estimates of $C_{\mathrm{sn}}$ at the five time points were: $2.65,2.21,2.33,2.46$ and $3.20 \%$ of binder. This gave a sample mean of 2.55 and sample standard deviation 0.179 .

The $D_{\text {eff }}$ averaged around $3 \times 10^{-13} \mathrm{~m}^{2} \mathrm{~s}^{-1}$ or around $10 \mathrm{~mm}^{2}$ year ${ }^{-1}$. Figure 4 shows a fitted line plot of $\log _{10}\left(\mathrm{TD}_{\text {eff }}\right)$ versus $\log _{10} \mathrm{t}$ where $\mathrm{T}($ tera $)=10^{12}$. The fact that the points vary around a straight line is some justification for the model $\mathrm{D}(\mathrm{t})=a \mathrm{t}^{\mathrm{n}}$. However, the points are clustered in pairs indicating the errors around the line are not independent. A similar cluster pattern was seen for other concrete mixes being studied in the same experiment $[5$, p.30], so the clustering is probably related to the weather, for example the proportion of time during which the blocks were splashed with seawater. To 
correctly model $\mathrm{D}_{\text {eff }}$ values we need to use a model such as

$$
\log _{10}\left(T D_{\text {eff }, i k}\right)=-0.4352-0.3115 \log _{10}(t)+e_{i}+e_{i k},
$$

where $e_{i}$ is a random effect for time $i$, and $e_{i k}$ is an independent random effect for replicate $\mathrm{k}$ at that time. We obtain estimated standard deviations $\operatorname{SD}\left(e_{i}\right)=0.036$ and $\operatorname{SD}\left(e_{i k}\right)=0.060$.

One of the $\mathrm{D}_{\text {eff }}$ figures at month 60 appears considerably lower than the other two, giving rise to the suspicion that it is erroneous. However, this point is not an extreme outlier compared to other concrete mixes in the same experiment and so we have chosen to leave it in the analysis. The danger of removing an outlier is that we might seriously underestimate the variance and hence overestimate the $95 \%$ lower confidence bound. A referee has suggested a curve that might be a better fit for these data than a straight line, but a test for curvature was not statistically significant $(\mathrm{P}$-value $=0.104)$, so we proceed with the standard power-law assumption [5, pp.9,39].

\section{Obtaining confidence bounds by simulation}

Confidence bounds for the lifetimes were obtained using a parametric bootstrap as follows. A grid of $x$ and $t$ values was chosen. For each $x$ and $t$ combination, the uncertainty in $\mathrm{C}_{\mathrm{sn}}$ was modelled by simulating a value from the Normal distribution with mean 2.55 and standard deviation 0.179 , while the uncertainty in the value of $\mathrm{D}(\mathrm{t})$ was modelled by simulating random errors $e_{i}$ and $e_{i k}$ from the Normal distributions with mean zero and standard deviations 0.036 and 0.060 respectively and substituting them in (7). Based on these values, values of $\mathrm{C}(x, t)$ were calculated using the industry standard model (4) and exact model (6). This was carried out 10000 times for each $x$ and $t$ combination, and the sample proportion of simulations that gave $C(x, t) \leqslant 0.5 \%$ was ascertained. Denote this sample proportion 
TABLE 1: Estimated lifetime $\mathrm{T}$ (years) using exact model until concentration $\geqslant 0.5 \%$ of binder for given cover with confidence Level.

\begin{tabular}{crrrr}
\hline Cover $\mathrm{mm}$ & $50 \%$ & $75 \%$ & $90 \%$ & $95 \%$ \\
\hline 20 & 17.3 & 16.3 & 15.4 & 14.8 \\
25 & 29.4 & 27.0 & 24.5 & 22.8 \\
30 & 49.6 & 42.8 & 38.0 & 35.6 \\
35 & 77.7 & 64.9 & 56.1 & 51.1 \\
40 & 114.4 & 92.9 & 78.6 & 71.4 \\
45 & 161.0 & 127.9 & 106.6 & 95.8 \\
50 & 218.1 & 169.9 & 139.5 & 124.3 \\
\hline
\end{tabular}

by $\hat{p}=\hat{p}(x, t)$. For fixed $x$, some analysis showed that a logit transformation of the sample proportions gave points that deviated only slowly from a straight line in time:

$$
\log \left(\frac{\hat{\mathrm{p}}}{1-\hat{\mathrm{p}}}\right) \approx \beta_{0}+\beta_{1} \mathrm{t} .
$$

This meant we could use linear interpolation between successive time points on the grid to obtain an adequate estimate of $\mathrm{T}$ corresponding to any desired confidence levels (such as $95 \%$ or $75 \%$ ). In Figure 5 the circles and solid line indicate the median time at which $C(x, t)=0.5 \%$ of binder, for various levels of cover $x$. This is practically the same as the deterministic solution using $C_{\mathrm{sn}}=2.55$ and $\log _{10}(\mathrm{TD})=-0.4352-0.3115 \log _{10} \mathrm{t}$ in the exact model (6). When more confidence is required, the curve moves progressively towards the left. The triangles indicate the $95 \%$ lower confidence bounds, meaning that for $95 \%$ of simulations the $\mathrm{C}(x, t) \leqslant 0.5 \%$ so the lifetime of the concrete was not exceeded. Numerical values of the confidence bounds are shown in Table 1 for the exact model. Table 2 shows the corresponding lower confidence bounds using the industry model (4), and the percentage by which the lifetime is overestimated compared to the exact model. Calculations for $0.4 \%$ binder are available from the authors. 


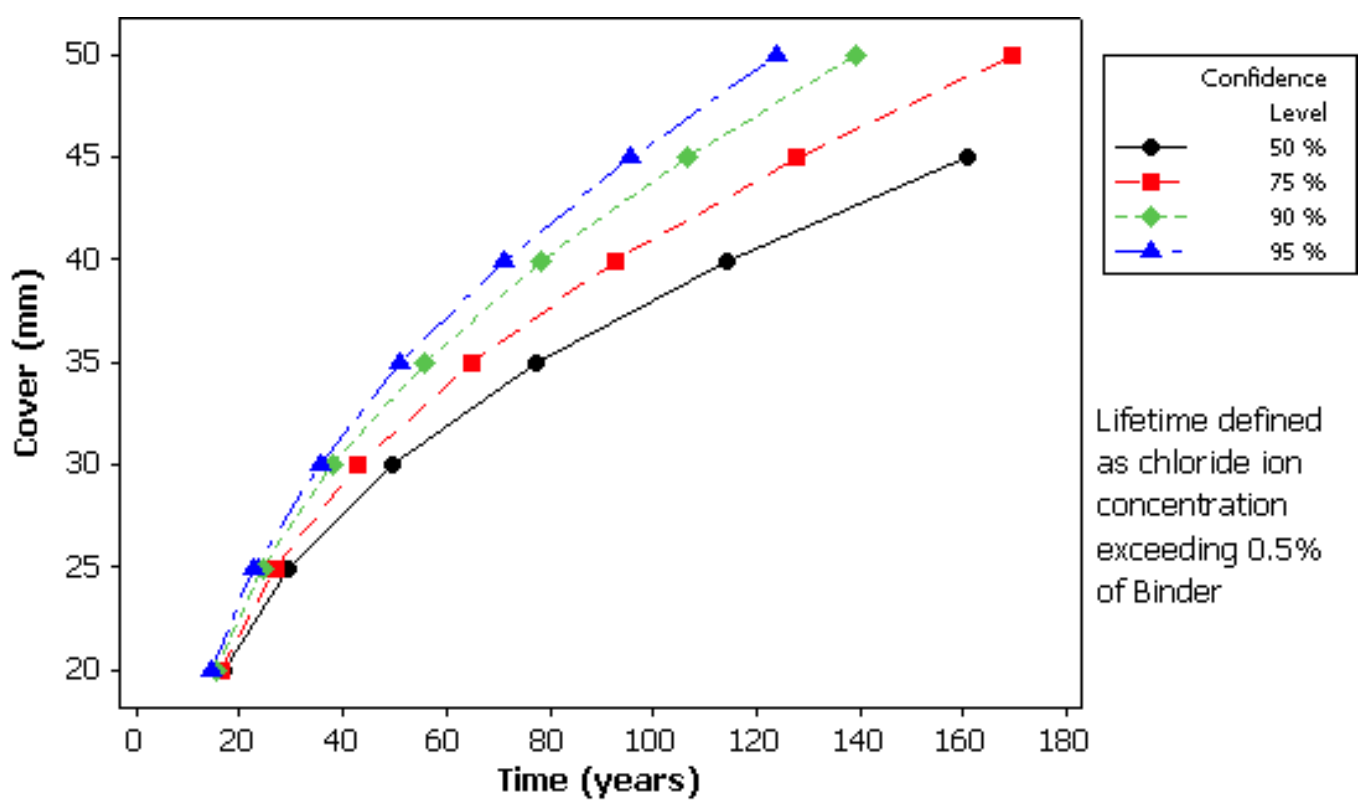

FIGURE 5: lower confidence bounds for concrete lifetimes with given cover, using $0.5 \%$ binder criterion and exact model. The lifetime predicted with stated confidence level to exceed the bound shown.

TABLE 2: Estimated lifetime $T$ (years) by usual industry model until concentration $\geqslant 0.5 \%$ of binder for given cover with confidence level (percentage by which lifetime is overestimated compared to exact model).

\begin{tabular}{c|rc|rc|rr|rr}
\hline Cover mm & $50 \%$ & $(\%)$ & $75 \%$ & $(\%)$ & $90 \%$ & $(\%)$ & $95 \%$ & $(\%)$ \\
\hline 20 & 27.5 & $(59)$ & 22.5 & $(38)$ & 19.0 & $(24)$ & 17.2 & $(16)$ \\
25 & 51.4 & $(75)$ & 40.0 & $(48)$ & 33.8 & $(38)$ & 29.8 & $(31)$ \\
30 & 87.4 & $(76)$ & 66.2 & $(55)$ & 53.1 & $(40)$ & 47.1 & $(32)$ \\
35 & 136.6 & $(76)$ & 99.7 & $(54)$ & 78.6 & $(40)$ & 68.6 & $(34)$ \\
40 & 199.4 & $(74)$ & 143.7 & $(55)$ & 110.2 & $(40)$ & 95.7 & $(34)$ \\
45 & 279.5 & $(74)$ & 197.6 & $(55)$ & 149.4 & $(40)$ & 128.1 & $(34)$ \\
50 & 378.0 & $(73)$ & 261.0 & $(54)$ & 195.7 & $(40)$ & 166.5 & $(34)$ \\
\hline
\end{tabular}




\section{Discussion}

By comparison with the exact model, the numerical results suggest that the usual formula (4) has a risk of overstating the effective lifetime of concrete, by over $70 \%$ for median lifetime and over $30 \%$ at the $95 \%$ lower confidence bound. On the other hand the exact model relies on the specific functional form of $\mathrm{D}(\mathrm{t})$ being correct and our experimental data are from a very short time scale. Therefore it is not yet possible to tell on purely pragmatic grounds which of the two models (the standard model with its ad hoc modification, or the exact model) will give the most accurate prediction for real concrete.

The scientific contribution of this article is that it derives a new formula for concrete industry researchers to use, and also illustrates how one can use the uncertainty in the parameter estimates to simulate concentration values $C(x, t)$ and thereby obtain lower confidence bounds for the concrete lifetimes.

Acknowledgements The data illustrated in this article were provided by the Building Research Association of New Zealand.

\section{A Proof of exact formula}

In what follows it is convenient to reparameterize $D(t)=a t^{n}$ as $D_{0}\left(t_{0} / t\right)^{\alpha}$ where $t_{0}$ is some reference time, $\alpha=-n$ (so $0 \leqslant \alpha<1$ ) and $a=D_{0} t_{0}^{\alpha}$. Fick's differential equation (5) therefore becomes

$$
\frac{\partial C}{\partial t}=D_{0}\left(\frac{t_{0}}{t}\right)^{\alpha} \frac{\partial^{2} C}{\partial x^{2}}, \quad \text { where } x>0 \text { and } t>0 .
$$

We need three initial conditions as follows. First, for any depth $x>0$ the concentration at time zero is assumed to be zero; that is, $\mathrm{C}(x, 0)=0$ (strictly 
speaking the differential equation is modelling the additional ions in excess of any background concentration $C_{i}$ ). Second, the concentration at the surface $x=0$ is assumed to be constant, independent of time. This is not exactly true in our study, but neither is $C_{\text {sn }}$ related to $t$ in any way that can be included in the differential equation: rather it is taken to vary randomly around its mean. Third, for any given time $t$, the concentration at depth $x$, $C(x, t) \rightarrow 0$ as $x \rightarrow \infty$ (the deeper the ions, the fewer).

Now try a transformed variable $z=t^{-\beta} x$ for some unknown $\beta$ to be found, and we write $C(x, t)=f(z)$ as a similarity solution. Now

$$
\frac{\partial^{2} C}{\partial x^{2}}=t^{-2 \beta} f^{\prime \prime}(z) \text { and } \frac{\partial C}{\partial t}=-\beta t^{-\beta-1} x f^{\prime}(z) .
$$

Therefore the diffusion model becomes

$$
-\beta t^{-\beta-1} x f^{\prime}(z)=D_{0}\left(\frac{t_{0}}{t}\right)^{\alpha} t^{-2 \beta} f^{\prime \prime}(z)
$$

and hence

$$
D_{0} t_{0}^{\alpha} f^{\prime \prime}(z)=-\beta x t^{-\beta-1+2 \beta+\alpha} f^{\prime}(z)=-\beta t^{\alpha+2 \beta-1}\left(x t^{-\beta}\right) f^{\prime}(z)=-\beta z f^{\prime}(z)
$$

provided that we choose $\alpha+2 \beta-1=0$. This implies $\beta=(1-\alpha) / 2$ which is $>0$ as expected (compare the classical solution with $\alpha=0$ ). So the solution satisfies $D_{0} t_{0}^{\alpha} f^{\prime \prime}(z)=-\beta z f^{\prime}(z)$, which implies

$$
f^{\prime \prime}(z)=\left(\frac{-\beta}{D_{0} t_{0}^{\alpha}}\right) z f^{\prime}(z), \quad \text { and hence } f^{\prime}(z)=A \exp \left(\frac{-\beta}{D_{0} t_{0}^{\alpha}} \frac{z^{2}}{2}\right),
$$

where $A$ is some constant. Hence

$$
f(z)=(-A) \int_{u=z}^{\infty} \exp \left(\frac{-\beta}{D_{0} t_{0}^{\alpha}} \frac{u^{2}}{2}\right) d u+B
$$

where $u$ is a dummy variable of integration and $B$ is an integration constant. Using a change of variables $w=\sqrt{2 \mathrm{D}_{0} t_{0}^{\alpha} / \beta} u$ one obtains $\operatorname{erfc}(w)$ and hence

$$
C(x, t)=f(z)=\left(C_{s n}-C_{i}\right) \operatorname{erfc}\left(\frac{\sqrt{\beta} z}{\sqrt{2 D_{0} t_{0}^{\alpha}}}\right)+C_{i}
$$


after relabelling the constants in terms of the chloride concentrations $\mathrm{C}_{\mathrm{sn}}$ and $C_{i}$. Finally, (6) follows after substituting $\beta=(1-\alpha) / 2=(1+n) / 2$ and $z=x / t^{\beta}=x / \sqrt{t^{1+n}}$.

\section{References}

[1] T. Siemes, R. Polder, H. De Vries. Design of concrete structures for durability. HERON, 43, 1998, 227-244. C410

[2] R. M. Ferreira. Probability based durability analysis of concrete structures in marine environment. $\mathrm{PhD}$ thesis, University of Minho, 2004. http://repositorium.sdum. uminho.pt/bitstream/1822/ 2675/1/PhDThesis(MiguelFerreira).pdf C412

[3] P. B. Bamforth. The derivation of input data for modeling chloride ingress from eight-year UK coastal exposure trials. Magazine of Concrete Research, 51, 1999, 87-96. C412

[4] G. S. Williamson, R. E. Weyers, M. C. Brown, A. Raminceanu, M. M. Sprinkel. Validation of probability-based chloride-induced corrosion service-life model.ACI Materials Journal, 105 (4), 2008, 375-380. C412

[5] N. P. Lee and D. H. Chisholm. Durability of Reinforced Concrete Structures under Marine Exposure in New Zealand. BRANZ Study Report SR145, 2005.

http://www.branz.co.nz/cms_show_download.php?id=123 C413, C417, C418

\section{Author addresses}

1. Barry McDonald, Institute of Information and Mathematical Sciences, Massey University, New Zealand. 
mailto:B.McDonald@massey .ac.nz

2. Graeme Hastie, Materials Technology Group, GDH Pty Ltd. Sydney, Australia.

mailto: Graeme. Hastie@ghd.com.au

3. Graeme Wake, Institute of Information and Mathematical Sciences, Massey University, NEw ZEALAND.

mailto:G.C.Wake@massey . ac.nz 